\title{
Multiplets in Emission of Large Quantum Dots in Microcavities
}

\author{
Fabrice P. Laussy, Alexey Kavokin, and Guillaume Malpuech \\ Lasmea, CNRS, Université Blaise Pascal - Clermont-Ferrand II \\ 24 avenue des Landais, 63177 Aubière Cedex, France
}

(Dated: November 21, 2018)

\begin{abstract}
We show theoretically that the emission spectrum of a single large quantum dot strongly coupled to a single photon mode in a microcavity can be qualitatively different from the spectrum obtained with an atom in a cavity. Instead of the well-known Mollow triplet we predict appearance of multiplets with the number of peaks a function of the quantum dot size and pumping intensity. The mutiplets can appear if the quantum dot is larger than the exciton Bohr radius, so that excitons are confined as whole particles in the dot. In this case the Pauli principle is relaxed and one can accommodate more than one exciton (but still a finite number) in a given quantum state.
\end{abstract}

PACS numbers: 42.50.Ct, 78.67.Hc, 42.50.Pq 
Introduction - Coupling of a zero-dimensional photonic mode to a zero-dimensional exciton (electron-hole) state has been realized in pillar and photonic microcavities containing quantum dots [1, 2, 3, 4]. Very recently, two groups have obtained independently the experimental evidence for vacuum-field Rabi splitting in such structures [3, 4]. These observations confirmed theoretical predictions of strong exciton-photon coupling in zero-dimensional systems [5, 6].

Nonlinear optical effects in strongly coupled zero-dimensional systems are expected to be extremely rich. For a while, only a manifestation of the Purcell effect [7] had been experimentally reported [1, 2], while theoretical predictions went much beyond, evoking appearance of dressed exciton and bi-excitons states [8]. The crucial question for description of emission from quantum dot cavities (QDC) is whether crystal excitations coupled to light behave like fermions or like bosons in these systems [9]. The fermionic regime has been a subject of cavity quantum electrodynamics (QED) for decades. It is familiar in atomic cavities, and can be efficiently described by Dicke formalism [10]. Its signature is the appearance of the Mollow triplet [11] in emission spectra of the cavity.

Clearly, the fermionic regime is realized if carriers are strongly confined in small-size quantum dots (case of [4], for example). On the other hand, it does not hold if the dot size is much larger than the exciton Bohr radius (further referred to as the "large dot" case, realized in particular in the dots originated from islands of quantum well width fluctuation [3, 12]). In large dots, excitons are quantized as whole particles and exhibit bosonic properties at least to some extent. The limiting case of a large dot is a quantum well where quasi-Bose condensation of excitons is indeed possible [13] and Rabi-doublets are observed in the spectra of microcavities [14]. Dicke formalism does not hold in this case, as it does not take into account the fundamental difference between an atomic and an excitonic system: the number of atoms is fixed while the number of excitons is not. From a formal point of view in the purely fermionic case the light mode is coupled to a two-level system, while in the purely bosonic case it is coupled to a harmonic oscillator having an infinite number of equidistant energy levels. Logically, the straightforward intermediate case would correspond to the optical mode coupled to a truncated harmonic oscillator, having a finite number, say $N$, of energy levels. As such, a large QD offers a realization of parastatistics which interpolate between Bose-Eistein and Fermi-Dirac statistics by allowing up to $N>1$ particles in a given quantum state [15, 16$]$. 
In this work we study theoretically the emission of light by a QDC using the model of a truncated harmonic oscillator. We assume that a finite number of excitons $(N \geq 1)$ can be generated in the quantum state of interest. Here $N$ is taken as a free parameter, expected to scale like $S_{\mathrm{dot}} / \pi a_{\mathrm{B}}^{2}$ where $S_{\mathrm{dot}}$ is the cross-section surface of the dot and $a_{\mathrm{B}}$ the exciton Bohrradius. Physically, it corresponds to the following image: the dot is much larger than the exciton Bohr radius, so that excitons are confined as whole particles inside it. $N=1$ recovers the fermionic case. We also assume that the energy separation between quantum confined excitonic states exceeds the vacuum Rabi splitting, so that one can neglect all excitonic states except one, strongly coupled to a single light mode. This latter condition, though very artificial for quantum well microcavities, is easily met in QDC, where typical values of the Rabi splitting are tens of $\mu \mathrm{eV}$ [3, 4]. Furthermore, we neglect exciton-exciton interactions, which allows us to assume the energy of the excitonic transition to be independent on the number $m$ of excitons already created in a given quantum state if $m<N$. If $m=N$ no more excitons can be created in the quantum state we consider. Finally, we assume the exciton-photon system to be fully spin-polarized, so that polarization effects can be neglected and bi-excitons bound state impossible. Experimentally this corresponds to the resonant circularly polarised pumping and no efficient spin-relaxation time in the system. In general to deal with QDC one should solve the complicated many-particles problem accounting for exciton-exciton, exciton-carrier and exciton-photon interactions. Here we deal with the simplified model described above as an ideal limit of more refined microscopic calculations [9] which display quantitative departures but an overall qualitative agreement. Our present model retains the essential physical difference of a large quantum dot from a small one and a quantum well and allows to obtain analytically the emission spectrum of the structure. We show that this spectrum is remarkably different from well-known Mollowtriplet and Rabi-doublet. It exhibits multiplets whose number is sensitive to the dot size and pumping intensity.

Formalism - The electromagnetic field is modelled in the approximation of the quasimode coupling by single mode Bose field annihilation operator $a$. We introduce for a large quantum dot, able to host $N$ excitons, the operator $\sigma_{N}$ which behaves like a Bose field annihilation operator when the number of excitations is less than or equal to $N$, i.e.,

$$
\sigma_{N}|m\rangle=\sqrt{m}|m-1\rangle, \quad \sigma_{N}^{\dagger}|m\rangle=\sqrt{m+1}|m+1\rangle \theta_{m, N}
$$


where $\theta_{m, N}$ is the modified Heaviside step function which is zero if $m \geq N$ and one if $m<N$. Its matrix representation in the basis of Fock states is the $N+1$ squared matrix which is a truncation of $\sigma_{\infty}$ the annihilation matrix of a Bose field. For instance, for $N=2$,

$$
\sigma_{2}=\left(\begin{array}{ccc}
0 & 1 & 0 \\
0 & 0 & \sqrt{2} \\
0 & 0 & 0
\end{array}\right) .
$$

The algebra for this operator follows straightforwardly:

$$
\left[\sigma_{N}, \sigma_{N}^{\dagger}\right]=\mathbf{1}_{N+1}-\lambda_{N+1}
$$

where $\mathbf{1}_{N+1}$ is the $N+1$ squared identity matrix and $\left(\lambda_{N+1}\right)_{i, j}=(N+1) \delta_{i, j} \delta_{i, N}$ (with $0 \leq$ $i, j \leq N)$.

The hamiltonian for our system is similar to Dicke [10] or Jaynes-Cummings [17] hamiltonian but instead of coupling the field $a$ with a spinor $J$ or a two-level system (fermion) $\sigma_{1}$, one couples $a$ with this operator $\sigma_{N}$, also in the rotating wave approximation:

$$
\begin{aligned}
H & =\hbar \omega a^{\dagger} a+\hbar \omega \sigma_{N}^{\dagger} \sigma_{N} \\
& +\hbar g\left(a^{\dagger} \sigma_{N}+a \sigma_{N}^{\dagger}\right) .
\end{aligned}
$$

We considered zero detuning for simplicity. The general case with a small detuning brings fine quantitative departures which are the topic for a separate communication. Here $\hbar g$ is the coupling constant corresponding to (half) the Rabi splitting in the linear regime [3, 4]. First line (3a) describes bare states of photons and excitons, the later being limited to a maximum $N$. We note eigenstates of this first line alone $|n, m\rangle$ with $n$ the number of photons, any integer number, and $m$ the number of excitons, any integer less than or equal to $N$. Second line (3b describes the coupling between the two fields which in absence of dissipation will always be "strong" in the quantum mechanical sense. The dissipation is caused by two channels of energy escape included as non-hermitian contributions of the Lindblad type to Von Neumann equation,

$$
i \hbar \dot{\rho}=[H, \rho]+\mathcal{L}_{a} \rho+\mathcal{L}_{\sigma_{N}} \rho,
$$

where

$$
\mathcal{L}_{A} \equiv-\frac{\gamma_{A}}{2}\left(A^{\dagger} A \rho-2 A \rho A^{\dagger}+\rho A^{\dagger} A\right)
$$


for $A=a\left(\right.$ resp. $\left.\sigma_{N}\right)$ describing the photon leakage through the cavity mirror by tunnel effect (resp. the emission from the active state within the cavity) with associated decay constant $\gamma_{a}$ (resp. $\gamma_{\sigma_{N}}$ ). The procedure is straightforward in principle: since the number of excitations is conserved by $H$, one diagonalises it in the subspace of $n$ excitations and thus obtains new eigenstates of the system (so-called dressed states in cavity QED terminology). Thus, in the basis $\mathcal{H}_{n}=\{|0, n\rangle,|1, n-1\rangle, \ldots,|N, n-N\rangle\}$ (for $n \geq N$ ), the hamiltonian reduces to:

$$
\begin{aligned}
H & =\hbar \omega n \mathbf{1}_{N+1} \\
& +\hbar g\left[\sqrt{i(n-i+1)} \delta_{i, j+1}\right. \\
& \left.+\sqrt{(i+1)(n-i)} \delta_{i, j-1}\right]_{0 \leq i, j \leq N}
\end{aligned}
$$

where the term between square brackets is the generic expression for the interaction matrix (which is zero everywhere but below and above its diagonal). Case $N=1$ gives the Mollow triplet.

Along with general results, we will deal explicitely with the all-important case $N=2$. It is expected the effect we predict will first be observed for this very value $N=2$. In this case, the matrix representation (6) in the basis $\mathcal{H}_{2}$ reads for $n$ excitations (with $n \geq 2$ )

$$
H=\hbar \omega n \mathbf{1}_{3}+\hbar g\left(\begin{array}{ccc}
0 & \sqrt{n} & 0 \\
\sqrt{n} & 0 & \sqrt{2(n-1)} \\
0 & \sqrt{2(n-1)} & 0
\end{array}\right),
$$

Cases where $n$ is lower than or equal to $N$ fall into linear regime displaying two peaks, the exciton field never reaching its saturation density. If $n>N$, the new $N+1$ eigenstates (dressed states) will be denoted, for the manifold $n$, by $|\nu\rangle_{n}$ with $\nu=1, \ldots, N+1$ indexing the state. For $N=2$,

$$
\begin{aligned}
& |\nu=1\rangle_{n} \equiv\left(\sqrt{\frac{n}{2(3 n-2)}},-\frac{\sqrt{2}}{2}, \sqrt{\frac{n-1}{3 n-2}}\right), \\
& |\nu=2\rangle_{n} \equiv\left(-\sqrt{\frac{2(n-1)}{3 n-2}}, 0, \sqrt{\frac{n}{3 n-2}}\right), \\
& |\nu=3\rangle_{n} \equiv\left(\sqrt{\frac{n}{2(3 n-2)}}, \frac{\sqrt{2}}{2}, \sqrt{\frac{n-1}{3 n-2}}\right) .
\end{aligned}
$$

in the bare states basis $\mathcal{H}_{2}$. The associated eigenvalues are:

$$
E_{\nu}^{n} \equiv \hbar \omega n+(\nu-2) \hbar g \sqrt{3 n-2}
$$


The spectrum of emission comes from transitions between manifolds with $n$ and $n-1$ excitations. One can obtain the exact expression for the luminescence spectrum from (4) and quantum regression theorem to evaluate the time correlation function needed for WienerKhintchin theorem. We content here with Fermi's golden rule formula for perturbations responsible for (5). These perturbations arise from the weak coupling of cavity photons $a$ (resp. excitons $\sigma_{N}$ ) with the continuum of external modes of the electromagnetic field in vacuum state. Therefore, calling $c_{i}^{\nu, n}$ the projection of state $|\alpha\rangle_{n}$ on state $|i, n-i\rangle$ one can obtain the coefficients in (8) as:

$$
|\nu\rangle_{n}=\sum_{i=0}^{N} c_{i}^{\nu, n}|i, n-i\rangle
$$

The corresponding transition probabilities between states $|\nu, n\rangle$ and $\left|\nu^{\prime}, n-1\right\rangle$ are obtained as:

$$
\begin{aligned}
& I_{\text {end }}=\left|\left\langle\left.\nu^{\prime}\right|_{n-1} a \mid \nu\right\rangle_{n}\right|^{2}=\left|\sum_{i=0}^{N}\left(c_{i}^{\nu^{\prime}, n-1}\right)^{*} c_{i}^{\nu, n} \sqrt{n-i}\right|^{2} \\
& I_{\text {lat }}=\left|\left\langle\left.\nu^{\prime}\right|_{n-1} \sigma_{N} \mid \nu\right\rangle_{n}\right|^{2}=\left|\sum_{i=1}^{N}\left(c_{i-1}^{\nu^{\prime}, n-1}\right)^{*} c_{i}^{\nu, n} \sqrt{i}\right|^{2}
\end{aligned}
$$

In cavity QED terminology, $I_{\text {end }}$ and $I_{\text {lat }}$ correspond to end-emission and lateral-emission photo-detection respectively, while in luminescence of a microcavity, one observes the sum of the two contributions $I_{\text {lat }}+I_{\text {end }}$ simultaneously. To detect $I_{\text {lat }}$ independently one should use the scattering geometry.

Results - Our main result is the appearance of multiplets in the configuration $n \geq N$. This is in stark contrast with cavity QED where nonlinearity results in apparition of the Mollow triplet [1]]). Three questions need be solved in the general case: first the number of peaks allowed by the transitions which is of a combinatorial nature, providing the number of transitions between two multiplets taking into account their degeneracy. Second the energy splitting between the various peaks thus obtained which is the eigenvalues problem. Third the strength of transitions which is the eigenvectors problem. If the radiative broadening is so small that all peaks can be discriminated and if no selection rules forbid some transitions, the multiplets structure is that of $2 N+1$ groups of more or less closely packed peaks for a total number of $(N+1)^{2}$. We label $\mu=0$ the group at the centre of the spectrum, and $\mu= \pm 1, \pm 2, \ldots$ groups located symmetrically about the central one. The number of 
peaks is $N+1-|\mu|$ in the $\mu^{\text {th }}$ group. Each peak is identified unambiguously by a couple of integers $(k, l)$ with $0 \leq k, l \leq N$. Peak $(k, l)$ belongs to group $\mu=k-l$ and is the $k^{\text {th }}$ (resp. $l^{\text {th }}$ ) if located at the left (resp. right) of the spectrum, counting as first in each group that of lower energy. The energy of this peak $E(k, l)$ is given by the photon energy released in the transition between two manifolds and is therefore given by the difference of initial and final eigenvalues $E_{k}^{n}$ in the dressed states basis:

$$
E(k, l)=E_{k}^{n}-E_{l}^{n-1}
$$

In the case $N=2$, from (9) and (12) we get in good approximation (the better the higher $n$ ) the mean energy of peak $(k, l)$ as

$$
E(k, l) \approx \hbar \omega+(k-l) \hbar g \sqrt{3 n}-(2 k-5 l+6) \frac{\hbar g}{2 \sqrt{3 n}}
$$

This gives the structure of the multiplet: there is a splitting of magnitude $|\nu| \hbar g \sqrt{3 n}$ between the central $(\mu=0)$ and the $\nu$ th group of peaks. The central group has a fine structure splitting (between $(1,1)$ and $(3,3))$ of $\sqrt{3} \hbar g / \sqrt{n}$. At the onset of the nonlinear effect $(n=3)$ the splitting is close to the Rabi splitting $2 \sqrt{(\hbar g)^{2}-\left(\gamma_{a}-\gamma_{\sigma_{2}}\right)^{2} / 16}$. The fine structure splitting of the groups $\mu= \pm 2$ is half the central one: $\sqrt{3} \hbar g /(2 \sqrt{n})$. As $n$ increases, each group of peaks gets farther from the others while inside this group, the fine structure splitting is reduced. This behaviour is seen in Fig. (II). When the photon field is so intense that for the given broadening $\gamma_{\sigma_{2}}$ the fine splitting is unobservable, the spectra resemble a Mollow triplet. It can be distinguished from it by the splitting which is $\sqrt{3} / 2$ times smaller and the presence of additional (small) peaks with a splitting $\sqrt{3}$ larger. Also the ratio between the central peak and its closest satellites is $9 / 4$ rather than $1 / 2$ in case of the Mollow triplet (see Fig. (10)).

This structure applies both for $I_{\text {end }}$ and $I_{\text {lat }}$ spectra. However, they display two drastically different behaviours. First $I_{\text {end }}$ is maximum at the center of the spectrum with peak $(2,2)$ the highest one. The situation is opposite with $I_{\text {lat }}$ for which, whatever $n$, peak $(2,2)$ is an exactly forbidden transition.

The same procedure can be applied to larger values of $N$, e.g., for $N=3$ (cf. 9):

$$
E_{\nu}^{n}=\hbar \omega n+\operatorname{sign}\left(\nu-\frac{5}{2}\right) \hbar g \sqrt{(-1)^{\nu} \sqrt{2} \sqrt{3 n^{2}-9 n+8}+3 n+4}
$$



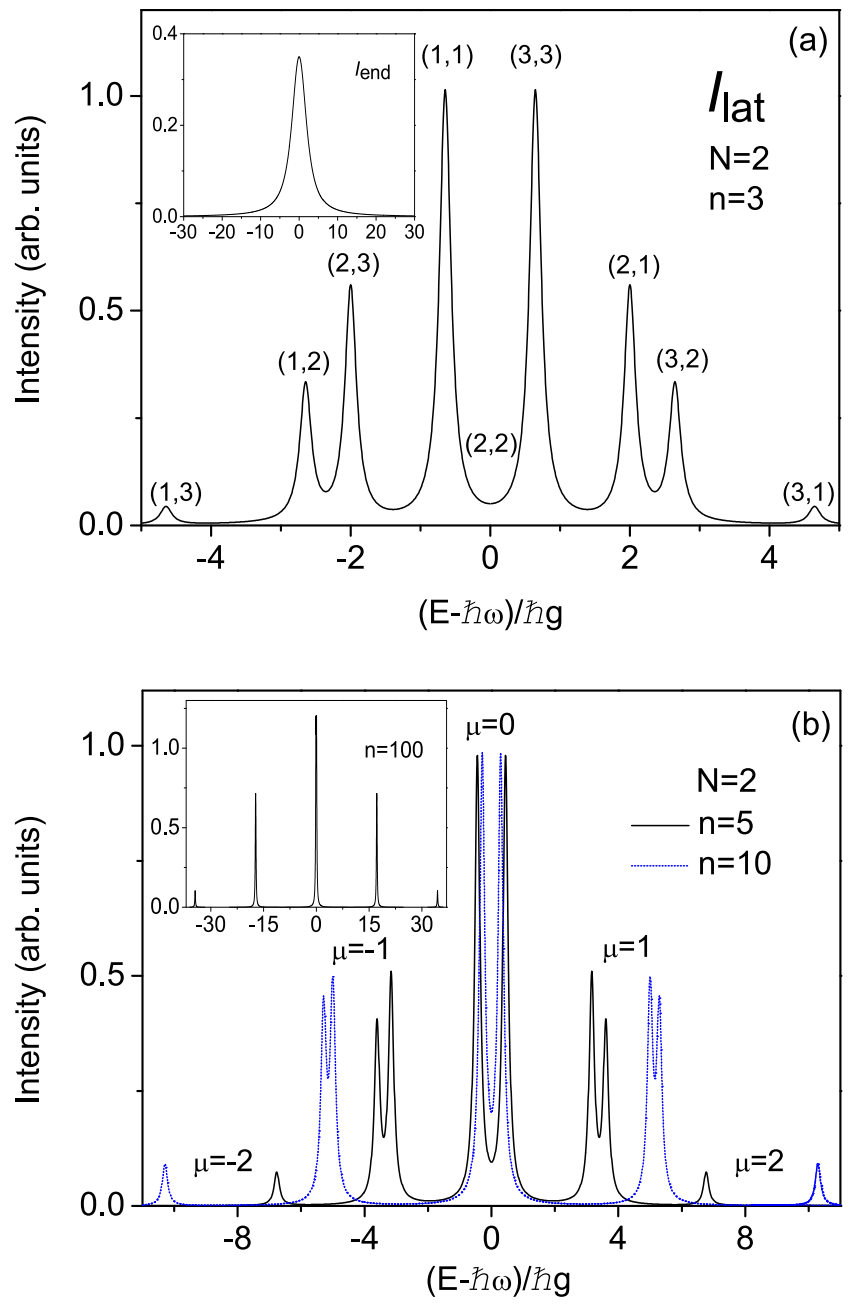

FIG. 1: Multiplets observed in the spectrum $I_{\text {lat }}$ of lateral emission of the cavity for (a) $N=2$ and $n=3$ and (b) $n=5$ (solid) and $n=10$ (dashed) (also for $N=2$ ). The coupling constant $\hbar g=$ $50 \mu \mathrm{eV}$. Broadening is obtained by convolution with Lorentz functions of width $\gamma_{a}=100 \mu \mathrm{eV}$ and $\gamma_{\sigma_{2}}=5 \mu \mathrm{eV}$ 3]. The inset in Fig. (a) showns $I_{\text {end }}$ for $n=3$, which is a Lorentz function; the inset of Fig. (b) shows $I_{\text {lat }}$ in the limit of huge $n(n=100)$.

with $1 \leq \nu \leq 4$, and for $N=4$ :

$$
\begin{aligned}
E_{\nu}^{n} & =\hbar n \omega+\operatorname{sign}(\nu-3) \hbar g \times \\
& \quad \sqrt{\operatorname{sign}(3-\nu)(-1)^{\nu} \sqrt{2} \sqrt{5 n^{2}-25 n+38}+5 n-10},
\end{aligned}
$$

this time with $1 \leq \nu \leq 5$, etc... (where $\operatorname{sign}(x)=0$ if $x=0$ and is $x /|x|$ otherwise.) Along the same lines as before, one can derive the structure and splittings of the spectrum of emission in these cases. For instance, for $N=3$, the $(1,1)-(3,3)$ splitting 

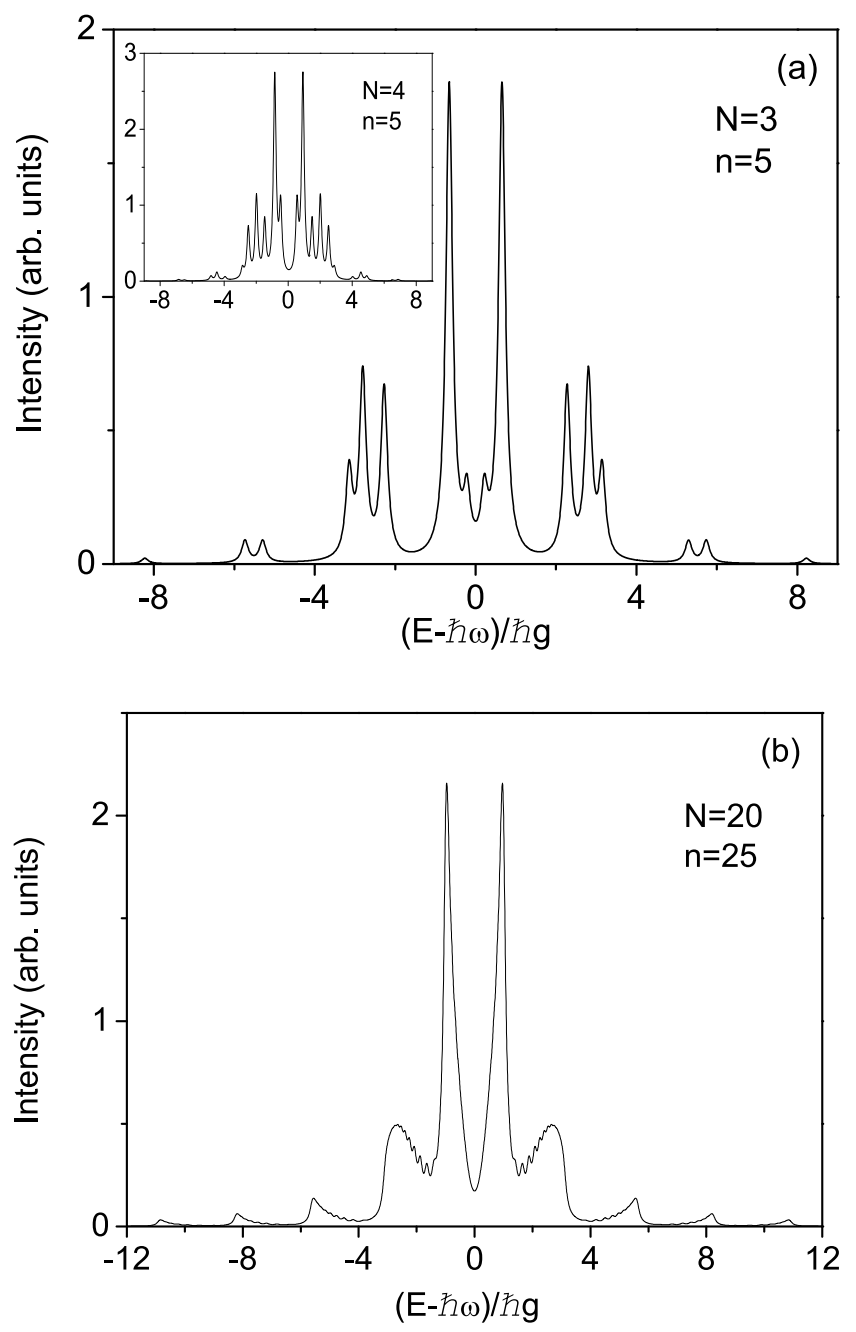

FIG. 2: Multiplets for (a) $N=3, n=5$ (inset $N=4, n=5$ ) and (b) $N=20, n=25$ with same parameters as in Fig. (11).

is $(\sqrt{3-\sqrt{6}}) \hbar g / \sqrt{n} \approx 0.74 \hbar g / \sqrt{n}$. Note that the difference in energy enters as square roots inside a square root and thus becomes smaller and smaller with increasing $N$. Figure (2) shows the case of an extremely large dot where satellite peaks become suppressed and the Rabi-like doublet dominates the spectrum. For $N \rightarrow \infty$ the familiar Rabi-doublet seen in the spectra of quantum well cavities is recovered by this model.

Conclusions - We predicted appearance of multiplets in emission spectra of zerodimensional photonic cavities containing single large quantum dots. The effect comes from relaxation of the Pauli principle for excitons confined as whole particles in large dots. The effect is strictly nonlinear as it requires the number of photons injected in the cavity to 
exceed the capacity of the dot. This capacity governs the number of peaks in emission. In the small dot limit, it reduces to three (Mollow triplets).

Acknowledgements - We thank Dr. Ivan Shelykh for his thorough discussion of our formalism. This work has been supported by the Clermont-2 project MRTN-CT-2003S03677.

[1] J. M. Gérard, B. Sermage, B. Gayral, B. Legrand, E. Costard, and V. Thierry-Mieg, Phys. Rev. Lett. 81, 1110 (1998).

[2] G. S. Solomon, M. Pelton, and Y. Yamamoto, Phys. Rev. Lett. 86, 3903 (2000).

[3] J. P. Reithmaier, G. Sek, A. Löffler, C. Hofmann, S. Kuhn, S. Reitzenstein, L. V. Keldysh, V. D. Kulakovskii, T. L. Reinecker, and A. Forchel, Nature 432, 197 (2004).

[4] T. Yoshie, A. Scherer, J. Heindrickson, G. Khitrova, H. M. Gibbs, G. Rupper, C. Ell, O. B. Shchekin, and D. G. Deppe, Nature 432, 200 (2004).

[5] L. C. Andreani, G. Panzarini, and J.-M. Gérard, Phys. Rev. B 60, 13276 (1999).

[6] M. A. Kaliteevski, S. Brand, R. A. Abram, V. V. Nikolaev, M. V. Maximov, C. M. S. Torres, and A. V. Kavokin, Phys. Rev. B 64 (2001).

[7] E. M. Purcell, Phys. Rev. 69, 681 (1946).

[8] G. Panzarini, L. C. Andreani, A. Armitage, D. Baxter, M. S. Skolnick, V. N. Astratov, J. S. Roberts, A. V. Kavokin, M. R. Vladimirova, and M. A. Kaliteevski, Phys. Rev. B 59, 5082 (1999).

[9] M. M. Glazov and F. P. Laussy, As yet unpublished (2005).

[10] R. H. Dicke, Phys. Rev. 93, 99 (1954).

[11] R. B. Mollow, Phys. Rev. 188, 1969 (1969).

[12] D. Gammon, E. S. Snow, B. V. Shanabrook, D. S. Katzer, and D. Park, Phys. Rev. Lett. 76, 3005 (1996).

[13] Y. E. Lozovik and V. I. Yudson, Pis'ma Zh. Eksp. Teor. Fiz. 22, 26 (1975), [JETP Lett. 22, 26 (1975).

[14] A. Kavokin and G. Malpuech, Cavity Polaritons, vol. 32 of Thin Films and Nanostructures (Elsevier, 2003).

[15] G. Gentile, Nuovo Cimento 17, 493 (1940). 
[16] H. S. Green, Phys. Rev. 90, 270 (1952).

[17] E. T. Jaynes and F. W. Cummings, Proc IEEE 51, 89 (1963). 